\title{
Coordinated development of business processes and their support systems
}

Traditional RE for business applications mainly concerns creating requirements for a software system based on business needs. For the past ten years, requirements on functionality of business applications have been slowly changing. The shift consists of moving from the command-based applications to the applications of workflow and groupware type. The difference between the traditional applications and the new generation is that while the former supports the execution of certain activities, e.g., to write a letter, to print an invoice, to complete a transaction, the latter supports the whole business process (BP). A business process support (BPS) system provides the possibility to run a business in a new, process-oriented way, a way which is more effective and efficient but which is not possible to introduce without a BPS system. This, in turn, demands to consider not only the requirements the business imposes on the system, but also the requirements the system imposes on the business. In the broader context, the focus of the development project should be shifted from the software development to coordinated development of business processes and BPS systems.

The goal of a co-development project can be formulated as creating and maintaining the fit between business processes and support systems. This implies a parallel evolution of the business and its BPS system as a reaction to changes in the business environment as well as to "revolutions" in the information technology. While the need for alignment of business processes and their support systems has been emphasized and discussed, there is a great need for systematic approaches and tools for achieving it.

G. Regev ( $\square)$

Ecole Polytechnique Federale de Lausanne (EPFL),

School of Computer and Communication Sciences,

1015 Lausanne, Switzerland

E-mail: gil.regev@epfl.ch

P. Soffer

MIS Department, Haifa University, Carmel Mountain,

31905 Haifa, Israel

I. Bider

IbisSoft AB, Box 19567, 10432 Stockholm, Sweden
The aim of this special issue is to fill the gap between the practical needs of co-development and the state of the theory and methodology of systems development, in general, and requirements engineering, in particular. This issue is a follow-up of the discussions at the Fifth International Workshop on Business Process Modeling Development and Support (BPMDS'04, Riga, Latvia), co-organized by the guest editors of this special issue; see http://www.ibissoft.se/events/bpmds04/bpmds04.htm for details.

The five papers presented in this special issue were carefully selected from 13 papers initially submitted to it. The selected papers went through a number of revisions based on the reviews and guest editors' comments. We hope that the resulting set represents a step forward in the direction of filling the above-mentioned gap. Below, we shortly overview the papers presented in the special issue.

In order to properly discuss relationships between business processes and BPS systems we need to understand more clearly what a BPS system is, how it could be differentiated from, and how it is connected to all the other systems in the jungle of software that exists or might exist in an organization. The proper way of getting such understanding is by matching an organization as a whole (in all its richness) to a set of supporting tools and systems. This is what Jan L.G. Dietz and Antonia Albani describe in their paper. The analysis is done based on the $\Psi$-theory that differentiates three interrelated levels within organizations. These organizational levels require different kinds of computer support that should be integrated. Based on the suggested theory, the paper gives some recommendations for an overall strategy of support systems' design.

Maintaining the fit between business processes and support systems presumes that we can measure the level of fit (or misfit) between the two. Otherwise, how would we know when it is time to begin a redesign iteration to adjust the system to the processes or the processes to the system? Is it possible to measure the fit in a quantitative way, or can it be expressed in a qualitative way only? A significant portion of the submissions to this special issue addresses the topic of evaluating and assessing the fit 
between BP and BPS systems as a basis for change decisions. The approaches range from quantitative measurement to qualitative diagnostic.

The paper by Anne Etien and Colette Rolland suggests an approach to quantitative measuring of fitness. The approach is based on creating a mapping between a business model and a system model and then quantitatively evaluating some characteristics of this mapping. To make the approach as general as possible, two highlevel ontologically based modeling techniques were chosen for creating a mapping. To make the approach practically applicable, the paper explains how common modeling techniques can be mapped to the chosen highlevel models. The theory worked out in the paper can be used for classifying and explaining the types of misfits that can potentially exist between business processes and support systems.

In practice, the business and system models do not always exist, and when they do exist, they may differ from reality, which gives rise to the issue of capturing and describing the difference. This issue is covered in detail in the following two papers. The papers by Wil M.P. van der Aalst and Nikolaus Kleiner propose techniques for discovering the business process as reflected by the BPS system and analyzing the fit with the prescribed business process.

The paper by van der Aalst proposes two analysis approaches, both qualitative and quantitative. The qualitative approach first generates Petri-Net models from event logs of the considered BPS system and then uses Petri-Net reduction operations in order to analyze the differences between this model and the prescribed model. The quantitative approach computes fit metrics based on the comparison between the actual event log and the prescribed model. The paper by Kleiner takes a qualitative analysis approach comparing an actual event $\log$ with the prescribed business process defined with a UML activity diagram. It describes the use of the InterPoL tool in semi-automatically diagnosing misfit cases in order to inform the change decision process. It explores the feasibility of this approach in different organizational settings considering the characteristics of both the BP and the BPS systems.

The goal of the BPS systems as explored by the first four papers was assumed to be the operational support of business processes related to the main activity of an organization. The paper by Bala Ramesh et al. considers a different kind of BPS system, namely a system that supports a higher-level process of the business process change management. This system keeps track of why the current set of business processes is shaped in a certain way. The explanation on the current shape of the business processes can be given in two ways, synchronous and historical. A synchronous way is a mapping of current organizational goals and business environment to the elements of the process definition. A historical way is an explanation behind the decisions taken for each of the previous transformation (also called design rationale). Both synchronous and historical views are of value for BP redesign. The paper by Ramesh et al. suggests an approach to the maintenance and use of the synchronous and historical information related to the BP design. The approach has been implemented by augmenting an existing knowledge-based system with a representation of the business process context (goals and environment) and design rationale.

This special issue is the result of much work by many people. We would like to thank the Requirements Engineering Journal co-editor Peri Loucopoulos and his team, as well as the reviewers of this special issue, who did an excellent job helping the authors to improve the quality of their papers. 